\title{
ON THE HOMOLOGY OF THE
}

\section{HILBERT SCHEME OF POINTS IN THE PLANE}

Geir Ellingsrud' and Stein Arild Strømme ${ }^{2}$

1 Matematisk institutt, Universitetet $i$ Oslo, Blindern, Oslo 3, Norway

2 Matematisk institutt, Universitetet i Bergen, 5014 Bergen, Norway

Although several authors have been interested in the Hilbert scheme $H_{i l b}{ }^{d}\left(\mathbb{P}^{2}\right)$ parametrizing finite subschemes of length $d$ in the projective plane ( $[\mathrm{I} 1],[\mathrm{I} 2],[\mathrm{F} 1],[\mathrm{F} 2],[\mathrm{Br}]$ among others) not much is known about the topological properties of this space. The Picard group has been calculated ([F2]), and the homology groups of $\mathrm{Hilb}^{3}\left(\mathbb{P}^{2}\right)$ have been computed ([H]). In this paper we give a precise description of the additive structure of the homology of $H_{i l b}{ }^{d}\left(\mathbb{P}^{2}\right)$, applying the results of BirulaBialynicki ( $[\mathrm{B} 1],[\mathrm{B} 2]$ ) on the cellullar decompositions defined by a torus action to the natural action of a maximal torus of SL(3) on $H_{i l l} b^{d}\left(\mathbb{P}^{2}\right)$. A rather easy consequence of the fact that this action has finitely many fixpoints is that the cycle maps between the Chow groups and the homology groups are isomorphisms. In particular there is no odd homology, and the homology groups are all free. The main objective of this work is to compute their ranks: the Betti numbers of $H_{i l b}^{d}\left(\mathbb{P}^{2}\right)$.

As a byproduct of our method we get similar results on the homology of the punctual Hilbert scheme and of the Hilbert scheme of points in the affine plane. 
It. seens natural to generalize our results to any toric smooth surface. Fowever, we give the results only for the rational rulea surfaces $F_{n}$ with an indication of the necessary changes in the proces.

For simplicity we work over the field of complex numbers, but with an appropriate interpretation of the word "homology" our results remain valid over any base field.

$\S 1$

Let $\mathbb{Q}^{2}$ be the projective plane over $\mathbb{C}$. For any positive integer a, let $H_{i l b}\left(\mathbb{P}^{2}\right)$ denote the Hilbert scheme pararietrizing finite subschemes of $\mathbb{P}^{2}$ of length $d$. If $\mathbb{A}^{2}$ denotes the complement of a line in $\mathbb{P}^{2}$, let $H i l b^{d}\left(A^{2}\right)$ denote the open subscheme of $\mathrm{Hilb}^{\mathrm{d}}\left(\mathbb{P}^{2}\right)$ corresponding to subschemes with support in $\mathbb{A}^{2}$. Furthermore let $\operatorname{Hilb}^{\alpha}\left(\mathbb{A}^{2}, 0\right)$ be the closed subscheme of Fil $I b^{d}\left(A^{2}\right)$ parametrizing subschemes supported in the origin.

For any complex variety $x$, let $H_{\star}(x)$ be the Borel-Moore homology of $X$ (homology with locally finite supports). By the i-th Betti number $b_{i}(x)$ we shall mean the rank of the finitely generated abelian group $H_{i}(x)$. Let $x(x)=\left[(-1)^{i_{b}}(x)\right.$ be the Euler-Poincaré characteristic of $X$. As usual, $A_{*}(X)$ is the Chow group of $x$, and $c l: A_{\star}(X) \rightarrow H_{*}(X)$ is the cycle map (see [Fu] ch. 19.1).

If $m$ and $n$ are non-negative integers, let $P(m, n)$ denote the number of sequences $n \geqslant b_{0} \geqslant b_{1} \geqslant \ldots \geqslant b_{m}=0$ such that $\left[b_{i}=m\right.$. If $n \geqslant m$, then $P(m, n)=P(m)$, the number of partitions of $m$. Let $P(m, n)=0$ if $m$ or $n$ is negative. 
(1.1) Theorem. (i) Let $X$ derote one of the schemes Filod $\left(\mathbb{P}^{2}\right)$,

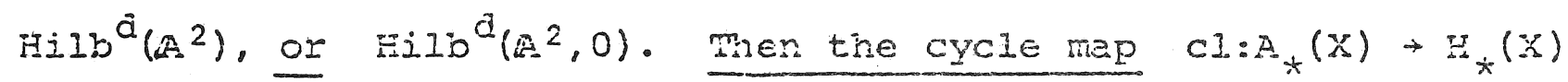
is an isomorphism, and in particular the ocd honology varishes.

Furthermore, both groups are Eree abelian groups.

(ii) $b_{2 k}\left(\right.$ Hilo $\left.D^{2}\left(p^{2}\right)\right)=a_{0}+d_{i}+d_{2}=a p+r=k-d_{1} p\left(p, d_{0}-p\right) p\left(d_{1}\right) p\left(2 a_{2}-r, r-d_{2}\right)$

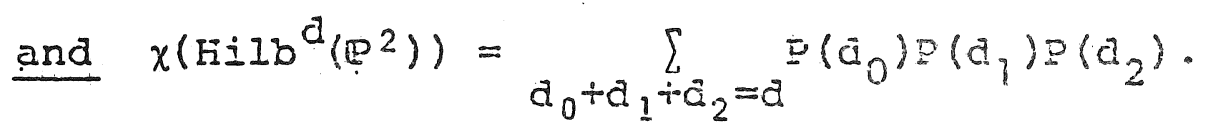

(iii) $b_{2 k}\left(H i l b^{d}\left(\mathbb{A}^{2}\right)\right)=P\left(2 d-k_{0} k-d\right)$ and $x\left(H i I b^{d}\left(A^{2}\right)\right)=P(d)$.

(iv) $b_{2 k}\left(H i I b^{d}\left(A^{2}, 0\right)\right)=P(k, a-k)$ and $x\left(H i b^{d}\left(A_{2}^{2}, 0\right)\right)=P(d)$.

Remark. The Betti numbers of $\mathrm{Hilb}^{3}\left(\mathbb{P}^{2}\right)$ were determined by $A$. Hirschowitz ([H]). In table 1 we have Iisted the Betti numbers of Hilbd $\left(\mathbb{R}^{2}\right)$ for $1 \leqslant a \leqslant 10$.

\begin{tabular}{|c|c|c|c|c|c|c|c|c|c|c|c|}
\hline 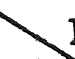 & 0 & 1 & 2 & 3 & 4 & 5 & 6 & 7 & 8 & 9 & 10 \\
\hline 1 & 1 & 1 & & & & & & & & & \\
\hline 2 & 1 & 2 & 3 & & & & & & & & \\
\hline 3 & 1 & 2 & 5 & 6 & & & & & & & \\
\hline 4 & 1 & 2 & 6 & 10 & 13 & & & & & & \\
\hline 5 & 1 & 2 & 6 & 12 & 21 & 24 & & & & & \\
\hline 6 & 1 & 2 & 6 & 13 & 26 & 39 & 47 & & & & \\
\hline 7 & 1 & 2 & 6 & 13 & 28 & 49 & 74 & 83 & & & \\
\hline 8 & 1 & 2 & 6 & 13 & 29 & 54 & 94 & 131 & 150 & & \\
\hline 9 & 1 & 2 & 6 & 13 & 29 & 56 & 105 & 167 & 232 & 257 & \\
\hline 10 & 1 & 2 & 6 & 13 & 29 & 57 & 110 & 189 & 298 & 395 & 140 \\
\hline
\end{tabular}

Table 1.

The Betti numbers $b_{2 k}\left(\operatorname{FiIb}^{\mathrm{d}}\left(\mathbb{L}^{2}\right)\right)$ are Iisted for $1 \leqslant d \leqslant 10$ and $0 \leqslant k \leqslant d$. For $d<k<2 d=d i m H_{i l o} d\left(\mathbb{P}^{2}\right)$ the number $b_{2 k}\left(H i l b^{d}\left(\mathbb{P}^{2}\right)\right)$ is given by Poincaré duality. 
(1.2) Corollary. (Briancon; [Br] V.3.3.) $\mathrm{HiIb}^{\mathrm{d}}\left(\mathrm{A}^{2} .0\right)$ is irrecucible.

Proof. By a result of Gaffney-Lazarsfeld (see [Ga] or [I2] theorern 2), any irreducible component of $\mathrm{Hilb}^{\mathrm{d}}\left(\mathrm{A}^{2}, 0\right)$ has ainension at least $d-1$. From (iv) of theorem (1.1) it follows that $b_{2 k}\left(E i l b^{d}\left(\mathbb{A}^{2}, 0\right)\right)=1$ if $k=d-1$ and 0 if $k>d-1$. The corollary folilows from [Fu] lemma 19.1 .1 .

Let $S$ denote the graded $z$-algebra freely generated by $c_{1}, \ldots, c_{d}, c_{1}^{i}, \ldots, c_{d}^{\prime}$ and $c_{2}^{\prime \prime}, \ldots, c_{d-1}^{\prime \prime}$ where the degree of $c_{i} c_{i}^{\prime}$ and $c_{i}^{\prime \prime}$ is $i$. Denote by $s_{k}$ the graded part of $s$ of degree $k$.

(1.3) Corollary. If $2 k<d$, then $b_{2 k}\left(H i I b^{d}\left(\mathbb{R}^{2}\right)\right)=r k_{Z} S_{k}$.

Proof. Assume $2 k \leqslant d$. Let $d_{0}, a_{1}, a_{2}, p$ and $r$ be indices such that the corresponding term in the expression for $b_{2 k}\left(H_{i} b^{d}\left(\mathbb{P}^{2}\right)\right)$ in (1.1) part (ii) is non-zero. Then $P\left(2 d_{2}-r_{1} r-d_{2}\right) \neq 0$ and $r-d_{2} \geqslant 0$. Therefore $p=k-d_{1}-r \leqslant k-d_{1}-d_{2}$ and hence $2 p \leqslant 2 k-2 a_{1}-2 d_{2} \leqslant d-2 d_{1}-2 d_{2} \leqslant a_{0}$. Thus $p \leqslant a_{0}-p$ and $p\left(p, a_{0}-p\right)=p(p)$. We may therefore write

$$
b_{2 k}\left(H i q b^{d}\left(\mathbb{p}^{2}\right)\right)=\sum_{p, a_{1}} p(p) p\left(d_{1}\right) B\left(k-d_{1}-p\right)
$$

where $B(j)=\sum_{m} P(2 m-j, j-m)$. This completes the proof since the Hilbert function of $z\left[c_{1}, c_{2}, \ldots\right]$ is $P(j)$, and that of $\mathscr{Z}\left[c_{2}, c_{3}, \ldots\right]$ is $B(j)$

The reason for giving this corollary is the following. Let $\pi: Z \longrightarrow \mathrm{Hilb}^{\mathrm{d}}\left(\mathbb{P}^{2}\right)$ be the universal family and let $\psi: Z \rightarrow \mathbb{P}^{2}$ be the 
natural map. Then $E_{i}=\pi_{*} \psi^{*} O_{\mathbb{P}^{2}}(i)$ are vectorbunales of rank $\bar{a}$ on Filb ${ }^{d}\left(P^{2}\right)$. The Chern classes of $E_{O}, E_{1}$ and $E_{2}$ are natural candiates for algebre generators of the Chow ring of fiilo ${ }^{2}\left(\mathbb{R}^{2}\right)$. One verifies that $c_{1}\left(E_{2}\right)=2 c_{1}\left(E_{1}\right)-a_{1}\left(E_{0}\right)$. The algebra $s$ therefore maps surjectively onto the subaigeire of $A^{*}\left(H_{i} I^{0}\left(\mathbb{R}^{2}\right)\right)$ generated by the Chern classes of the $E_{i}{ }^{\prime} s$. The coroilary can thus be regarded as evicience for the follovirg conjerture

(1.4) Conjecture. $A^{*}\left(H_{i} I b^{d}\left(R^{2}\right)\right)$ is generated as a $Z$-algebre by the Chern-classes of $E_{0}, E_{1}$ and $E_{2}$.

We end this section by recalling two results which are fundamental for this work.

Following Fulton ([Fu] example 1.9.1) we say that a schene $x$ has a cellullar decomoosition if there is a filtration $x=x_{n} \supset x_{n-1} \supset \ldots \supset x_{0} \supset x_{-1}=\emptyset$ by closed subscheres with each $x_{i}-X_{i-1}$ a disjoint union of schemes $U_{i j}$ isomorphic to affine spaces $A^{n_{i j}}$. The U $U_{i j}$ 's will be called the cells of the decomposition.

(1.5) Proposition. Let $x$ be a scheme with a cellullar decomposition. Then for 0 sisdin $x$

(i) $\mathrm{H}_{2 \mathrm{i}+1}(\mathrm{X})=0$

(ii) $\mathrm{H}_{2 i}(\mathrm{X})$ is a $\mathrm{Z}$-moaule freely generated by the classes of the closures of the i-dimensional celis.

(iii) The cycle map cl: $x_{*}(X) \rightarrow E_{*}(X)$ is an isomorphism. 
For a proof of this proposition see [FU] chapter 19.1.

Let $X$ be a variety with an action of $G_{m}$ and let $x$ be a fixpoint. Then there is an induced action of $\mathbb{G}_{\mathrm{m}}$ on the tangent space $\mathrm{T}_{\mathrm{X}, \mathrm{X}}$. The part of $\mathrm{T}_{\mathrm{X}, \mathrm{x}}$ where the wieights of $\mathbb{E}_{\mathrm{m}}$ are positive is denoted by $\left(\mathrm{T}_{\mathrm{X}, \mathrm{X}}\right)^{+}$. The following theorem is proved in $[\mathrm{B} 1]$ and $[\mathrm{B} 2]$.

(1.6) Theorem. (Birula-Bialynicki). Let $x$ be a smooth projective variety with an action of $\mathbb{G}_{m_{i}}$ Suppose that the fixpoint set $\left\{x_{1} \ldots . . x_{n}\right\}$ is finite, and let $x_{i}=\left\{x \in X \mid \lim _{t \rightarrow 0} t x=x_{i}\right\}$. Then

(i) $x$ has a celluliar decomposition with cells $x_{i}$. (ii) $\mathrm{T}_{\mathrm{x}_{i}, \mathrm{x}_{i}}=\left(\mathrm{T}_{\mathrm{X}, \mathrm{x}_{i}}\right)^{+}$. 
$\$ 2$.

From now on we fix a system of homogeneous coorainates $T_{0}, T_{1}, T_{2}$ of $\mathbb{P}^{2}$. Let $G \subseteq S L(3, C)$ be the maximal torus consisting of all diagonal matrices. We denote by $\lambda_{0}, \lambda_{1}, \lambda_{2}$ the complex characters of $G$ such that for any $g \in G$ we have $s=\operatorname{diag}\left(\lambda_{0}(g), \lambda_{1}(g), \lambda_{2}(g)\right)$. Then $G$ acts on $\mathbb{P}^{2}$ via $g T_{i}=\lambda_{i}(g) T_{i}$, and on points $\left(a_{0}, a_{1}, a_{2}\right)$, this action is given by $g\left(a_{0}, a_{1}, a_{2}\right)=\left(\lambda_{0}(g)^{-1} a_{0}, \lambda_{1}(g)^{-1} a_{1}, \lambda_{2}(g)^{-1} a_{2}\right)$. The fixpoints are clearly $P_{0}=(1,0,0), P_{1}=(0,1,0)$ and $P_{2}=(0,0,1)$.

Let $L$ be the line $T_{2}=0$, and put $F_{0}=\left\{P_{0}\right\}, F_{1}=L-P_{0}$. and $F_{2}=\mathbb{P}^{2}-\mathrm{L}$. Then $F_{i}=\mathbb{A}^{i}$, and they define a cellullar decomposition of $\mathbb{P}^{2}$. The one-parameter subgroups $\phi: \mathbb{G}_{m} \rightarrow G$ inducing this cellullar decomposition are those of the type $\phi(t)=\operatorname{diag}\left(t^{w_{0}}, t^{w_{1}}, t^{w_{2}}\right)$ where $w_{0}<w_{1}<w_{2}$ and $w_{0}+w_{1}+w_{2}=0$.

The action of $G$ on $\mathbb{P}^{2}$ induces in a natural way an action of $G$ on $\operatorname{Hilb}^{d}\left(\mathbb{P}^{2}\right)$. If $z \subseteq \mathbb{P}^{2}$ corresponds to a fixpoint of this action, clearly the support of $z$ is contained in the fixpoint set $\left\{P_{0}, P_{1}, P_{2}\right\}$ of $G$. Hence we may write $z=z_{0} U z_{1} \cup z_{2}$ where $z_{i}$ is supported in $P_{i}$ and corresponds to a fixpoint in Hilb ${ }^{d}{ }\left(\mathbb{P}^{2}\right)$, where $a_{i}=\operatorname{length}\left(\mathrm{O}_{i}\right)$.

(2.1) Lemma. The action of $G$ on $\operatorname{Hilb}^{d}\left(\mathbb{P}^{2}\right)$ has only finitely many fixpoints.

Proof. A point of Hillb $^{d}\left(\mathbb{P}^{2}\right)$ is a fixpoint if and only if the corresponding ideal $I$ in $\mathbb{C}\left[T_{0}, T_{1}, T_{2}\right]$ is invariant under $G$, which is the case if and only if $I$ is generated by monomials. These ideais obviously form a finite family. 
It is weil known that $\mathrm{Kilb}^{\mathrm{d}}\left(\mathrm{E}^{2}\right)$ is smooth and projective ([Gr], [Fl]). Herce (1.5) and (1.6) apply to the action of any sufficiently general one-paraneter suogrovo of $G$ on $\mathrm{Hi}^{\mathrm{d}}\left(\mathbb{P}^{2}\right)$, anc we have proved the statements in (1.1) part (i) concerning Hilb $\left(\mathbb{P}^{2}\right)$. To prove the rest of $(1.1)$ it remains to count the ceils of a given dimension. For this purpose we use a decomposition of the Hilbert scheme wich we now procesd to ciescribe.

For any $z \subseteq \mathbb{P}^{2}$ of finite length d we can woite $z$ uniqueIy as a disjoint union $z=z_{0} \cup Z_{1} \cup Z_{2}$ where eaci $z_{i}$ is a closec subscheme of $\mathbb{P}^{2}$ supported in $F_{i}$. Put $\bar{c}_{i}(z)=$ length $\left(0_{z_{i}}\right)$. For any triple $\left(a_{0}, a_{1}, a_{2}\right)$ of non-negative integers with $d=a_{0}+\bar{a}_{1}+\bar{a}_{2}$, we define $w\left(d_{0}, \bar{d}_{1}, d_{2}\right)$ to be the (locally closed) subset of Fil $b^{d}\left(\mathbb{P}^{2}\right)$ corresponding to subschemes $z$ with $d_{i}(z)=a_{i}$ for

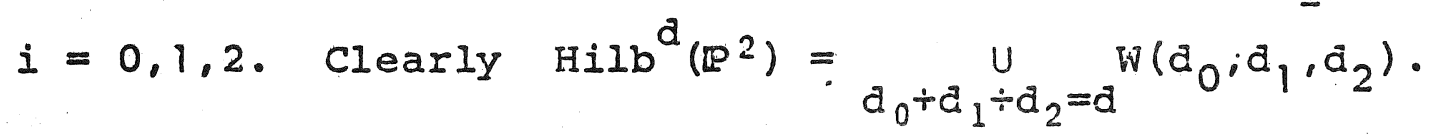

Let $\phi$ be any one-parameter subgroup of $G$ respecting the celluilar decomposition $\left\{F_{0}, F_{1}, F_{2}\right\}$ of $\mathbb{P}^{2}$. Then $\phi$ inauces a cellullar decomposition of $\operatorname{Hilb}^{d}\left(\mathbb{P}^{2}\right)$, and $w\left(a_{0}, a_{1}, a_{2}\right)$ is a union of cells from this decomposition. In fact, let $z$ be in $w\left(d_{0}, d_{1}, d_{2}\right)$ and write $z=z_{0} u z_{1} u z_{2}$. Then, as $t \rightarrow 0, \phi(t)\left(z_{i}\right)$ approaches, a subscheme supported in $\mathrm{P}_{\dot{I}}$. Thus $W\left(d_{0}, d_{1}, d_{2}\right)$ has a cellullar decomposition and $(1.5)$ applies to it.

since $w\left(d_{0}, a_{1}, d_{2}\right) \approx w\left(d_{0}, 0,0\right) \times w\left(0, d_{1}, 0\right) \times w\left(0,0, d_{2}\right)$ we get

$(2.2)$ Lemma. $i_{2 K}\left(\operatorname{HiIb}^{d}\left(\mathbb{E}^{2}\right)\right)=$ $a_{0}+d_{1}+d_{2}=d p+q+r=k b_{2 p}\left(w\left(a_{0}, 0,0\right)\right) b_{2 q}\left(w\left(0, a_{1}, 0\right)\right) b_{2 r}\left(w\left(0,0, a_{2}\right)\right)$.

This reduces our problem to the calculation of the Retti numbers oI $w\left(a_{0}, 0,0\right), w\left(0, a_{1}, 0\right)$ and $w\left(0,0, a_{2}\right)$. 
$\$ 3$.

The spaces $w(a, 0,0), W(0, a, 0)$ and $w(0,0, a)$ are all contained in Fis ${ }^{\mathrm{d}}\left(\mathbb{P}^{2}\right)$. In the previous section we saw that they are unions of cells from a cellullar decomposition of Hilb $\left[\mathbb{T P}^{2}\right)$. The cells contaired in $w(d, 0,0)$ (resp. $w(0, d, 0), w(0,0, a)$ ) are exactly those corresponaing to fixpoints supported in $P_{0}$ (resp. $P_{1}, P_{2}$ ). Wie are thus reduced to the study of G-invariant slitschemes of $\mathbb{R}^{2}$ concentrated in one fixpoint of $G$. Any such subschemes is contained in a G-invariant affine piane. Hence we are interested in ideais of $R=\mathbb{C}[x, y]$ of finite colength, invariant under the action of a two-dimensional torus $T$ given by $t_{\circ} x=\lambda(t) x$ and $t \cdot y=\mu(t) y$, where $\lambda$ and $\mu$ are two linearly indepencient characters of $T$. We shall also denote by $\lambda$ and $\mu$ the elements in the representation ring of $T$ induced by the corresponaing oneàimensional representations.

Let $I$ be such an ideal. Then since $I$ is T-invariant, it is generated by monomials in $x$ and $y$. Hence the numoer $b_{j}=i n f\left\{k \mid x j_{Y} k_{f I}\right\}$ exists for each integer $j \geqslant 0$. clearly $b_{j}=0$ if $j>>0$. Let $I$ be the least integer such that $b_{F}=0$. The $b_{j}$ form a non-increasing sequence and $\sum_{j=0}^{r} b_{j}=l e n g t h(R / I)=d$. Furthermore $v^{b_{0}}, x y^{b_{1}}, \ldots, x^{j_{y}} y^{b}, \ldots, x^{r}$ is a (not necessarily minimal) set of generators for $I$. Note that this sets up a one-one corresponaience between $T$-invariant ideals of colength $d$ in $R$ and partitions of $d$.

For any ordered pair $\underline{a}=(\alpha, \beta)$ of integers, let $R[\alpha, \beta]$, also denoted $R[\underline{a}]$, be the $R$-module $R$ with tre action of $T$ given by $t \cdot x^{m} y^{n}=\lambda(t)^{m-\alpha} \alpha_{\mu}(t)^{n-\beta_{X}{ }^{m} y^{n}}$. In the representation ring of $T$ we may write $R[\alpha, \beta]=\sum_{\substack{p \geqslant-\alpha \\ q \geqslant-\beta}} \lambda^{p_{\mu}}$. 
(3.1) Lemna. There is a T-equivariant cesolution

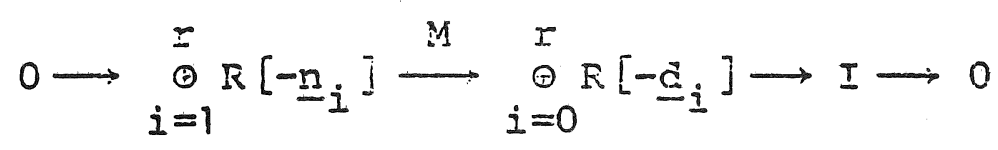

where $\underline{n}_{i}=\left(i, b_{i-1}\right)$ and $\underline{a}_{i}=\left(i, b_{i}\right)$. If $e_{i}=b_{i-1}-b_{i}$ for $1 \leqslant i \leqslant 1$ then

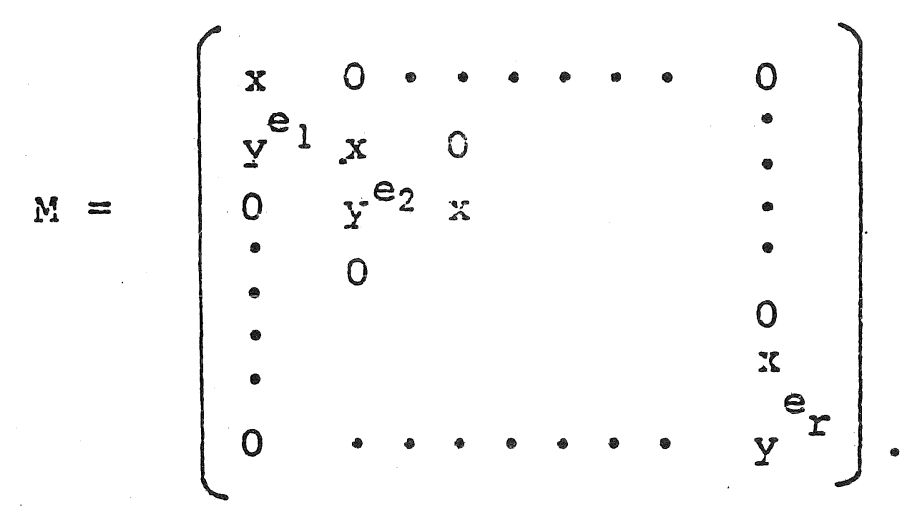

Proof. This amounts to checking that $M$ is equivariant and that

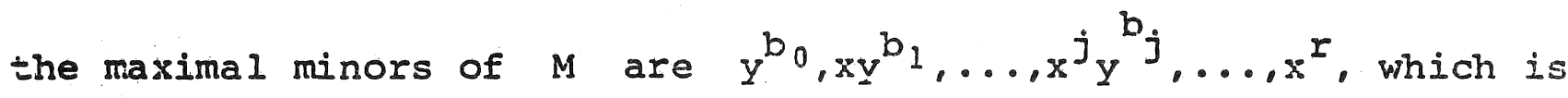
straight forwara.

(3.2) Lemma. In the representation ring of $T$ we have the identi$\underline{t y}$

$$
\operatorname{Hom}_{R}(I, R / I)=\sum_{1 \leqslant i<j<1} \sum_{s=b_{j}}^{b_{j+1}^{-1}}\left(\lambda^{i-j-1}{ }_{\mu}^{b_{i-1}-s-1}+\lambda^{j-i}{ }_{\mu}^{b_{i-1}-s}\right) .
$$

Proof. First we prove that $\operatorname{Fon}_{R}(I, R / I)=E x t_{R}^{l}(I, I)$ in a $T$-equivariant way. The T-equivariant exact sequence

$$
0 \rightarrow I \rightarrow R \rightarrow R / I \rightarrow 0
$$

induces a T-equivariant sequence

$$
0 \rightarrow \operatorname{Hom}_{R}(I, R / I) \longrightarrow \operatorname{Ext}_{R}^{l}(I, I) \rightarrow \operatorname{Ext}^{I}(I, R) \rightarrow \operatorname{Ext} I(I, R / I) \rightarrow 0
$$

The last map of this sequence is an isomorphism because 


$$
\operatorname{Ext}_{R}^{\frac{1}{R}}(I, R) \cong \operatorname{Ext}_{\frac{2}{2}}^{2}(R / I, R) \cong \operatorname{Ext}_{R}^{2}(R / I, R / I) \cong \operatorname{Ext}_{R}^{\frac{1}{R}}(I, R / I)
$$

To compute $\operatorname{Ext} \frac{1}{R}(I, I)$ we use the $T$ equivariant complex

$$
E_{0}^{v} \otimes E_{1} \stackrel{A}{\rightarrow}\left(E_{0}^{v}\left(B E_{0}\right) \vartheta\left(E_{1}^{v} Q E_{1}\right) \stackrel{B}{\rightarrow} E_{1}^{v} \otimes E_{0}\right.
$$

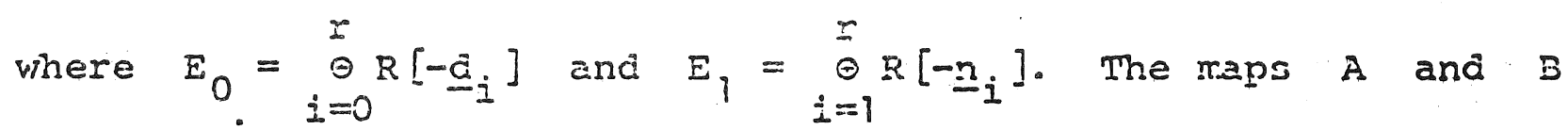

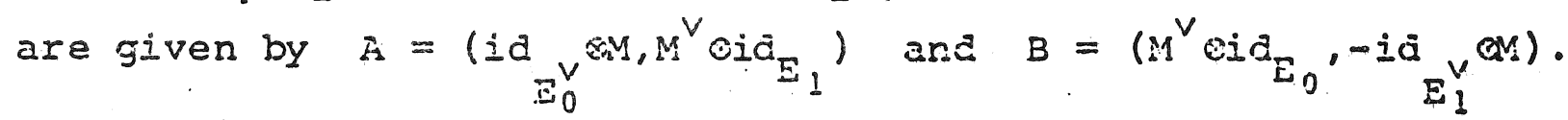
The cokernel of $B$ is $\operatorname{ext}_{R}(I, I)$, the middle honology is Hom $_{R}(I, I)=R$, and $A$ is injective. Hence in the representation ring we get the formula

$\operatorname{Ext}_{R}^{1}(I, I)=$

$R+\sum_{\substack{1<i<r \\ 0<j \leqslant r}} R\left[\underline{n}_{i}-\underline{a}_{j}\right]-\sum_{1 \leqslant i, j \leqslant r} R\left[\underline{n}_{i}-\underline{n}_{j}\right]-\sum_{0<i, j \leqslant r} R\left[\underline{a}_{i}-\underline{a}_{j}\right]+\sum_{\substack{1<i<r \\ 0<j \leqslant r}} R\left[\underline{a}_{j}-\underline{n}_{i}\right]$.

For $1<i \leqslant j \leqslant r$ define $K_{i j}=R\left[\underline{n}_{j}-\underline{d}_{i-1}\right]-R\left[\underline{n}_{i}-\underline{n}_{j}\right]-R\left[\underline{a}_{j}-\underline{a}_{i-1}\right]+R\left[\underline{a}_{j}-\underline{n}_{i}\right]$ and $I_{i j}=R\left[\underline{n}_{i}-\underline{a}_{j}\right]-R\left[\underline{n}_{i}-\underline{n}_{j}\right]-R\left[\underline{a}_{i-1}-\underline{a}_{j}\right]+R\left[\underline{a}_{i-1}-\underline{n}_{j}\right]$. Then, regrouping the terms in the formula above, it is easily verified that $\operatorname{Ext}_{R} \frac{1}{R}(I, I)=\sum_{1 \leqslant i \leqslant j \leqslant r} K_{i j}{ }^{i I}{ }_{i j}$. Now using that $a_{j}=\left(j, b_{j}\right)$ and that $\underline{n}_{i}=\left(i, b_{i-i}\right)$, we get

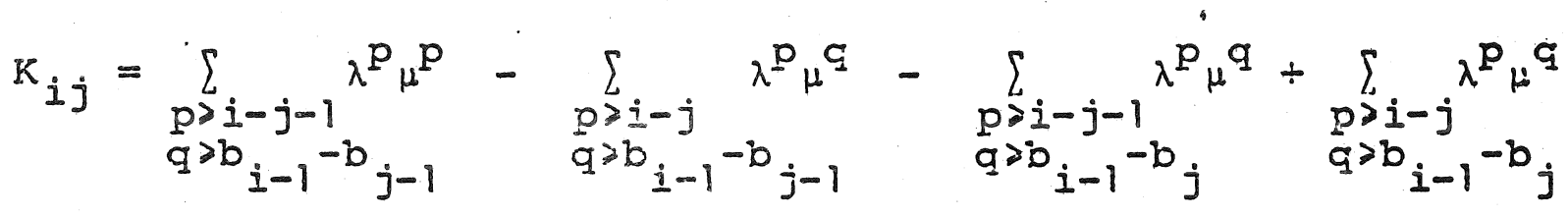

$$
\begin{aligned}
& =\sum_{q \geqslant b_{i-1}-b_{j-1}} \lambda^{i-j-1} \mu^{q}-\sum_{q \geqslant b_{i-1}-b_{j}} \lambda^{i-j-1} \mu^{q} \\
& =\sum_{s=b_{j}}^{b} \lambda^{i-j-1} b_{\mu-1}^{b_{i-1}-1} \text {. }
\end{aligned}
$$

In a similar way one checks that $I_{i j}=\sum_{s=b_{j}}^{b} \lambda^{j-1}{ }^{s-b_{i-1}}$. 
$\$ 4$

We ncri proceed to compute the Betti numbers of $w(0,0, d), w(0, d, 0)$ ard $w(a, 0,0)$. We start with $w(0,0, a)$.

As all the subschemes of $\mathbb{P}^{2}$ corresponding to points in $W(0,0, d)$ are contained in the affine plane spec $\mathbb{C}\left[\frac{T_{0}}{T_{2}}, \frac{T_{1}}{T_{2}}\right]$ we put $x=\frac{T_{0}}{T_{2}}$ and $y=\frac{T_{1}}{T_{2}}$. In the computation in $\S 3$ we may take $T=G$; then $\lambda=\lambda_{0} \lambda_{2}^{-1}$ ena $\mu=\lambda_{1} \lambda_{2}^{-1}$.

Choose a one-parameter sukgroup $\phi: \mathbb{F}_{\mathrm{m}} \neq \mathrm{G}$ given by $\phi(t)=\operatorname{ciag}\left(t^{w_{0}}, t^{w_{1}}, t^{w_{2}}\right)$ where $w_{0}<w_{1}<w_{2}$ and $w_{0}+w_{1}+w_{2}=0$. Then $\lambda \circ \phi(t)=t^{W_{0}{ }^{-W_{2}}}$ and $H_{0} \phi(t)=t^{W_{1}-W_{2}}$. More generaIIy, for any character $\lambda^{c}{ }_{\mu}^{\beta}$ oI $G$ we have $\lambda^{\alpha}{ }_{\mu}^{\beta}$ od $(t)=t^{\alpha\left(w_{0}-w_{2}\right)+\beta\left(w_{1}-w_{2}\right)}$.

Pick a cell $U$ from the cellullar decomposition of $H_{i l b}{ }^{d}\left(\mathbb{L}^{2}\right)$ desined by $\phi$, contained in $w(0,0, d)$. We want to compute its dimension. The cell U corresponas to a fixpoint of $G$ in $H_{i} 1 b^{d}\left(\mathbb{P}^{2}\right)$, contained in Spec $\mathbb{C}\left[\frac{T_{1}}{T_{2}}, \frac{T_{1}}{T_{2}}\right]=$ Spec $\mathbb{C}[x, y]$, hence to an invariant ideal $I$ in $\mathbb{C}[x, y]$. According to $(1.2)$, $\operatorname{dim} U=\operatorname{dim} T^{+}$where $T$ is the tangent space of $H_{i l b}{ }^{d}\left(\mathbb{P}^{2}\right)$ at the fixpoint. There is a canonical G-equivariant identificetion $T=\operatorname{Hom}_{R}(I, R / I)$ where $R=C[x, y]$ (see $[G r]$ ). We may assume that $\frac{w_{2}-w_{0}}{w_{1}-w_{1}}>>0$. Then any one aimensional representation $\lambda^{\alpha} \mu^{\beta}$ occurring in $\operatorname{Hom}_{R}(I, R / I)$ has a positive weight witin respect to $\phi$ if and only if $c<0$, or $\alpha=0$ and $\beta<0$. It follows from (3.2) that

$$
T^{+}=\sum_{1 \leqslant i \leqslant j \leqslant r} \sum_{s=b}^{b_{j-1}-1} \lambda^{i-j-1} \mu^{b_{i-1}-s-1}+\sum_{j=1}^{r} \sum_{s=b}^{b_{j-1}} \mu^{s-b_{j-1}} .
$$

The number of summands in the first sum is $\sum_{i=1}^{r} \sum_{j=i}^{r}\left(b_{j-1}-b_{j}\right)=$ $\sum_{i=1}^{r} b_{i-1}=a$ and in the second sum there are $\sum_{j=1}^{r}\left(b_{j-1}-b_{j}\right)=b_{0}$ summands. Therefore $\operatorname{dim} U=\mathrm{dim} \mathrm{T}^{+}=\mathrm{d}+\mathrm{b}_{0}$. 
In order to comoute one of the Eetti numbers of $w(0,0, d)$, say $D_{2 \mathrm{k}}(w(0,0, d))$, we kave to count the number of cells of aimension k. Since there is a one-cne correspondence between invariant ideals of $\mathbb{C}[\mathrm{x}, \mathrm{y}]$ of colength $\mathrm{a}$ and gartitions $b_{0}>b_{1}>\ldots>b_{L}=0$ of $d, b_{2 k}(w(0,0, d))$ is the number of partitions of $2 a-k$ in parts bounded by $k-d$. We have proved

(4.1) Proposition. $b_{2 k}(N(0,0, a))=P(2 d-k, k-d)$.

Remark. This concludes the proof of theoren (1.1) part (iii) since $W(0,0, d) \hat{=} H i 1 D^{d}\left(\mathbb{A}^{2}\right)$.

Next we turn to $w(a, 0,0)$. Subschemes of $\mathbb{p}^{2}$ corresponding to points in $W(d, 0,0)$ are supported in $P_{0}$. In particular they are contained in spec $\mathbb{C}\left[\frac{T_{1}}{T_{0}}, \frac{T_{2}}{T_{0}}\right]$. Put $x=\frac{T_{1}}{T_{0}}$ and $y=\frac{T_{2}}{T_{0}}$. In the computation in $\$ 2$ we may take $T=G_{1} \lambda=\lambda_{1} \lambda_{0}^{-1}$, and $\mu=\lambda_{2} \lambda_{0}^{-1}$. Choosing a one-parameter'subgroup $\phi$ ivith $w_{0}<w_{1}<w_{2}$ and $\frac{w_{1}{ }^{-w_{0}}}{w_{2} 2^{-w_{0}}}>>0$, and reasoning as above, we get

$$
T^{+}=\sum_{1 \leqslant i<j \leqslant \Sigma} \sum_{s=b_{j}}^{b-1} \lambda^{j-i}{ }^{s-b_{i-1}}
$$

where $T$ is the tangent space to $\mathrm{Hilb}^{\mathrm{d}}\left(\mathbb{Q}^{2}\right)$ at the fixpoint corresponding to the partition $b_{0} \geqslant b_{1} \geqslant \ldots>b_{r}=0$ of $d$. Wence the aimension of the corresponding cell is $\sum_{i=1}^{r} \sum_{j=i+1}^{r}\left(b_{j-1}-b_{j}\right)=$ $\sum_{i=1}^{r} b_{i}=a-b_{0}$. This gives

(4.2) Proposition. $\vdots_{2 k}(w(d, 0,0))=P(k, d-k)$. 
Remark. This proves theorem (1.1) part (ii) since $W(a, 0,0)=\operatorname{LiI}^{2}\left(\mathbb{A}^{2}, 0\right)$.

The last case to treat is $w(0, d, 0)$. This time we put $x=\frac{T_{0}}{T_{1}}$, $y=\frac{T_{2}}{T_{1}}, \lambda=\lambda_{0} \lambda_{1}^{-1}$, anc $\mu=\lambda_{2} \lambda_{1}^{-1}$.

As usual, let $\phi$ be a cre-parameter subgroup of $G$ with $w_{0}<w_{1}<v_{2}$. Let $\lambda_{H_{b}}^{\beta}$ be a one-aimensional representation of $G$ with $\alpha \beta \leqslant 0$. Since $w_{0}^{-w_{1}}<0$ and $w_{2}{ }^{-w_{1}}>0$ the weight of $\lambda_{. \mu}^{\alpha}$ with respect to $\phi$ is positive if and only if $\epsilon<0$ and $\beta>0$. Using this and (3.2) it is easily verified that

$$
T^{+}=\sum_{1 \leqslant i \leqslant j \leqslant r} \sum_{s=b}^{b} \lambda_{j}^{i-1^{-1}} \lambda^{i-j-1} b_{i-1}-s-1
$$

where $T$ is the tangent space of $\operatorname{Hilb}^{\mathrm{d}}\left(\mathbb{P}^{2}\right)$ at the fixpoint corresponding to the partition $b_{0} \geqslant b_{1} \geqslant \ldots \geqslant b_{r}=0$ of d. Hence all the cells in $W(0, d, 0)$ are of dimension $d$, and we get

(4.3) Proposition. $\quad b_{2 k}(w(0, d, 0))=\left\{\begin{array}{lll}0 & \text { if } & k \neq d \\ P(d) & \text { if } & k=d^{*}\end{array}\right.$

Substituting the expressions of $(4.1),(4.2)$ and $(4.3)$ in the formula in lemma (2.2) we get theorem (1.1) part (ii). This concludes the proof of $(1.1)$. 
8.5.

Denote by $\mathbb{F}_{n}$ the rational, ruled surface $\mathbb{P}\left(0_{\mathbb{P}} \mathbb{P}_{\mathbb{P}^{1}}(-\Omega)\right)$. I maximal torus $T$ of the automorphism group of $E_{n}$ is of dimension two and has four fixpoints on $E_{n}$. It is easily checked that for en appropriate class of one-parameter subgroups of T, the weights on the tangent space of $F_{n}$ at two of these Iixpoints are of opposite sign, anc at the two remaining fixpoints, the two weights are respectively positive and negative. Thus the corresponding cellullar decomposition of $E_{n}$ contains a point, two copies of $\mathbb{A}^{1}$, and an $\mathbb{A}^{2}$. Adapting the proof of (1.1) to this situation we get

(5.1) Theorem. The cycle map $c l: \mathbb{A}_{\star}\left(\operatorname{Hilb}^{\mathrm{d}}\left(\mathbb{F}_{n}\right)\right) \rightarrow \mathrm{F}_{\star}\left(\mathrm{Hilb}^{\bar{d}}\left(\mathbb{F}_{n}\right)\right)$ is an isomorphism, and in particular the odd homology vanishes. The homology groups are free abelian groups. Furthermore,

$$
\begin{aligned}
& b_{2 k}\left(\operatorname{Hilb}\left(E_{n}\right)\right)= \\
& a_{0}+a_{1}+a_{2}+a_{3}=a p+r=k-a_{1}-a_{2} p\left(p, a_{0}-p\right) p\left(a_{1}\right) P\left(a_{2}\right) p\left(2 a_{3}-r, r-a_{3}\right)
\end{aligned}
$$

and

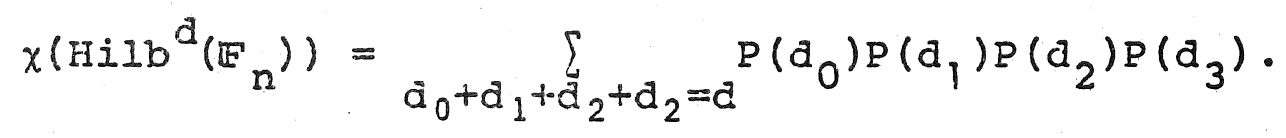


References.

[B1] Eialysicki-Birule, A.: Some theorems on actions of algerraic groups. Annals of Mathematics, Vol. 98, No. 3 (1973) pp $480-\div 97$.

[E2] Bialynicki-Birula, A.: Some Properties of the Decompositions cf Algebraic Varieties Determined by Actions of a Torus. Bullein ae l'Pcademie Polonaise des Sciences. Série des sciens math. astr. et phys. Vol 24, No. 9 (1976)

p. $667-674$.

[E1] Fogarty, J.: Algebraic families on an algebraic surface. Anex. T. Math. $10(1968) 511-521$.

[F'] Fogarty, J.: Algebraic families on in algebraic surface II: Picard scheme of the punctual Hilbert scheme. Amer. J. Math. 96 (1979) $660-687$.

[Br] Briarcon, J.: Description de $\mathrm{Hilb}^{\mathrm{n}_{\mathfrak{C}}}\{\mathrm{x}, \mathrm{y}\}$. Inventiones math. 41 (1977) 45-89.

[Fu] Fulton, W.: Intersection theory. Ergebnisse der Mathematik und ihrer Grenzgebiete. Springer-Verlag 1984.

[Ga] Gaffney, T.: Multiple points ana associated ramification loci. In Singularities, Proceedings of symposia in pure mathematics of the AMS. Volume 40 , part 1.

[Gr] Grothendieck. A.: Techniques de construction et théorèmes d'existence en sèometrie algébrique IV: Les schémas de Hilbert. Sem. Bourbaki 221 (1960/61).

[H] Hirschowitz, A.: Le group de Chow équivariant. C. R. Acad. Sc. Paris t 298 (1984) 87 .

[I1] Iarrobino, A.: Punctual Hilbert Schemes, Memoirs of the AMS, No 188 (1977).

[I2] Iarrobino, A.: Deforming complete intersection Artin algebras. In Singularities, Proceedings of symposia in pure mathematics of the AMS. Volume 40 , part 1. 